\title{
Scalp Metastasis of Cervical Cancer With Favorable Outcome
}

\author{
Elham Saffarieh $^{(\oplus)}$, Setare Nassiri $^{2 *}$, Maedeh Brahman $^{2}$, Soheila Amini Moghaddam² ${ }^{2}$ Shima Hosseini ${ }^{2}$
}

\begin{abstract}
Introduction: Carcinoma of the uterine cervix is the most common gynecological malignancies in developing countries. Human papilloma virus is known as the main etiology. In addition, the spread of uterine cervical cancer often occurs through direct local extension and the lymphatics although the hematogenous spread is uncommon. Further, the scalp metastasis of cervical cancer is extremely rare.

Case Presentation: In this regard, a 50-year-old woman with scalp metastasis of previous cervical cancer was discussed in the present study. She was suffering from a fast-growing and painful nodule, located on the frontal part of the scalp as a sole site of metastasis. A multidisciplinary approach was considered for her, which consisted of radical excision, followed by radiotherapy and chemotherapy. Given the lack of abundant evidence for the efficacy of this treatment, our patient has fortunately survived for more than two years. Conclusions: During the follow-up period after the completion of treatment, it is not sensible to only focus on the primary site of the tumor and thus entire examination is mandatory in this regard.

Keywords: Cervical cancer, Metastasis, Scalp
\end{abstract}

\section{Introduction}

Cervical cancer is one of the most common gynecologic malignanciesin developing countries. (1)Anoverwhelming majority of cervical tumors includes the squamous cell while adenocarcinoma and poorly differentiated cancers are observed as well. (2) Most cases are not metastatic at the moment of diagnosis due to symptoms such as postcoital bleeding, abnormal menstrual cycle, or foul smell discharge which compel the patient to refer for the examination leading to early diagnosis. The spreading of cervical cancer in these stages is via a direct metastatic way to the vagina, bladder, or rectum. Regional lymph node involvement is also possible. However, the distant metastasis of cervical cancer either in primary disease or the recurrence setting is rare and mostly observed in the lung, liver, and bone $(3,4)$. Furthermore, skin metastasis in cervical cancer is unusual. Among solid tumors, the skin involvement of breast cancer and gastrointestinal tumors are more common compared to the others (5). It should be noted that scalp metastasis is an extremely rare type of skin metastasis in cervical cancer, and in our review of the literature, only 8 cases have so far been reported. This exceptional situation resulted in horrible outcomes. This report introduced a patient with the scalp metastasis of cervical cancer with unbelievable survival rates after a combination approach including surgery, radiation, and chemotherapy.

\section{Case Presentation}

A 50-year-old woman, gravida 2, live child 2, referred to our center with painful swelling in the frontal region of the scalp (Figure 1). She was a known case of cervical cancer stage II $B$, who had received external beam radiation 28 courses and brachytherapy 3 courses, followed by 6 cycles of taxol and cisplatin. Moreover, she had a regular follow-up and was symptom-free for two years after the completion of chemoradiotherapy. At presentation, she did not have any vaginal or pelvic symptoms such as vaginal bleeding, vaginal discharge, and pelvic pain. There was a $3 \times 3 \mathrm{~cm}$ painful nodule, located on the frontal part of the scalp which was fast-growing although there was no more evidence of the recurrence in general examinations. As regards medical history, the patient mentioned that she had a surgery for the sebaceous cyst of the scalp about 20 years ago. Thus, it expressed our doubts about the infected sebaceous cyst as a common pathology in the female scalp. On the other hand, cervical cancer in her past history justified our crucial decisions of performing fineneedle aspiration biopsy. The metastatic squamous cell carcinoma of the cervix was established on microscopic examinations and immunohistochemistry staining. The existence of simultaneous metastasis in other sites was a matter of concern to us in such exceptional circumstances. Therefore, magnetite resolution imaging (MRI), and positron emission tomography/computed tomography (PET/CT) were performed accordingly (Figure 2). Both of 


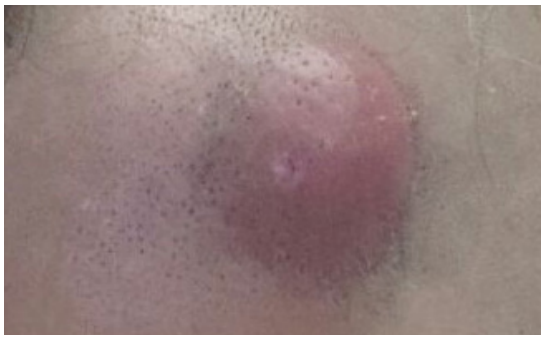

Figure 1. Painful Swelling on the Frontal Region of the Scalp due to Cervical Cancer Metastasis.

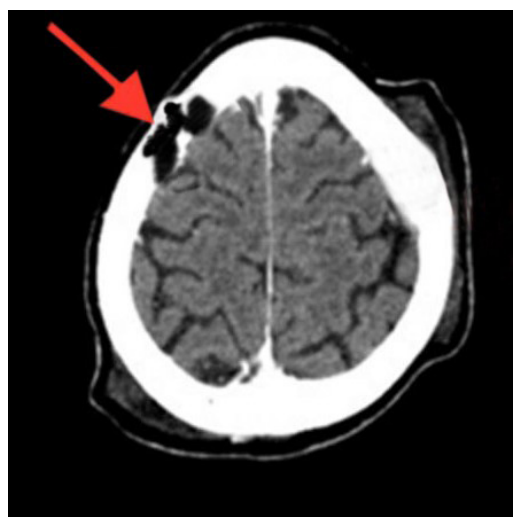

Figure 2. MRI of the Scalp. Note. MRI: Magnetite resolution imaging. The arrow shoves a swelling area which is located in the frontal part of the scalp.

them approved that there was only a sole site of metastasis. This unusual controversial condition of the disease posed a real challenge to our team to make a joint decision. Generally, the distant metastasis of cervical cancer leaves surgeons with no choice except palliative therapy. Thus, we resected the scalp nodule with wide local excision and achieved free of tumor margins. Then, we performed irradiation and chemotherapy. So far, she has been entirely symptom-free after 2 years. Based on our policy at Oncology Department, there is an informed consent form filled by the patient allowing us to use her information for the case report.

\section{Discussion}

The spread of uterine cervical cancer often occurs through direct local extensions and the lymphatics. Hematogenous spread is uncommon, and in such an unusual situation, the most frequently reported metastasis sites are lungs, bone, and liver. Skin metastasis is extremely rare in cervical cancer even in the late stage of the disease. In other words, the incidence of skin metastasis is $1-2 \%$ and the most common sites are abdominal wall, vulva, and chest. (6) Among skin metastases, scalp involvement is absolutely rare and has so far been reported only in seven cases. In most cases, there is simultaneous metastasis with it (7-10). Scalp metastasis as a sole site of involvement is reported only in one case (11). In general, the skin metastasis of cervical cancer, like other solid tumors, presents a single or multiple painful nodule which is not ulcerative. Accordingly, such an isolated complaint maybe a possible cause of missed-diagnosis as a benign and a more common condition. For instance, dermoid and sebaceous cysts are the usual non-malignant nodules in the scalp which can be even painful when there is a superimposed infection $(12,13)$. Occasionally, malignant tumors such as Kaposi's sarcoma and mycosis fungoid may be possible in the scalp even in patients with a previous history of cervical cancer as a secondary tumor which may arise due to immunodeficiency caused by primary cancer itself or chemo-radiation (14). The skin metastasis of cervical cancer can be single or multiple but they manifest as a painful nodule in all cases. In one case, it accompanied with alopecia (15). According to bed filtering vascular phenomena, the lung is the most common site for the distant hematological spreading of solid tumors. Moreover, lung involvement can be discovered as a single site or simultaneous with other sites metastasis while other metastases without lung involvement are rare. The exact mechanism of this strange behavior is yet unknown. However, the lymphatic obstruction and retrograde embolism of the tumoral cell is theoretically considerable (16). In our patient, there was no evidence of the distant metastasis of cervical cancer except for scalp involvement. Scalp metastasis as the sole site of involvement is extremely rare and is related to an undifferentiated tumor. Conversely, in our case, either primary tumor or distant metastasis was squamous cell carcinoma (large cell non-keratinizing). This type of pathology may be a natural cause of the good prognosis of our patient's disease. Another underlying cause of acceptable prognosis was the longer interval between primary disease and recurrence. Literature review proved that more prolonged interval is associated with better results. In most cases of the skin metastasis of cervical cancer after several months of treatment completion, the spreading of the tumor was discovered while, in our case, it appeared after about two years. The survival rate is low and something in the region of few weeks or months (17). However, our patient is, fortunately, alive after 3 years. As a matter of fact, scalp metastasis is usually accompanied with the other site of spreading the solid tumor thus PET/CT may be a logical plan for finding the subtle and small metastasis in addition to exact examinations. In our patient, PET/CT and MRI illustrated only a sole site involvement in the scalp. Palliative therapy is a unanimous agreement in this situation. The principal aim of palliation is to improve the quality of life instead of the overall survival rate. In the present study, combination therapy was performed for the patient and she survived contrary to popular belief. It was thought that she should take the chance to have a multidisciplinary approach although the brevity, acceptability, and safety of multidisciplinary treatment were crucial. Due to the sole site of metastasis, it 
was necessary to direct our attention to surgical excision. We did it successfully with free of tumor margins and then considered irradiation and chemotherapy. Nevertheless, we were waiting for an inevitable and tragic consequence. However, she has a good survival rate after three years of fallow-ups. Therefore, we decided to report this rare type of scalp metastasis of cervical cancer. In conclusion, we recommend that it is crucial to pay attention to the entire sites of the body while not just focusing on pelvic regions in dealing with cervical cancer during the follow-up. Each swelling on the skin may be evidence of the distant metastasis of the tumor and biopsy should be considered accordingly. With the earlier diagnosis of the sole site of metastasis, a favorable outcome may be achievable, along with performing combination therapy.

\section{Conflict of Interests}

Authors declare that they have no conflict of interests.

\section{Ethical Issues}

Informed consent was obtained from the patient for publication of this report.

\section{Financial Support}

None.

\section{References}

1. Schwartz RA. Cutaneous metastatic disease. J Am Acad Dermatol. 1995;33(2 Pt 1):161-182. doi:10.1016/0190-9622(95)90231-7

2. Mathew A, George PS. Trends in incidence and mortality rates of squamous cell carcinoma and adenocarcinoma of cervix--worldwide. Asian Pac J Cancer Prev. 2009;10(4):645-650.

3. Benoulaid M, Elkacemi H, Bourhafour I, et al. Skin metastases of cervical cancer: two case reports and review of the literature. J Med Case Rep. 2016;10(1):265. doi:10.1186/s13256-016-1042-0

4. Maheshwari GK, Baboo HA, Ashwathkumar R, Dave KS, Wadhwa MK. Scalp metastasis from squamous cell carcinoma of the cervix. Int J Gynecol Cancer. 2001;11(3):244-246. doi:10.1046/j.15251438.2001.00074.x

5. Brownstein $\mathrm{MH}$, Helwig EB. Metastatic tumors of the skin. Cancer. 1972;29(5):1298-1307. doi:10.1002/1097-0142(197205)29:5<1298::aidcncr2820290526>3.0.co;2-6

6. Hayes AG, Berry AD 3rd. Cutaneous metastasis from squamous cell carcinoma of the cervix. J Am Acad Dermatol. 1992;26(5 Pt 2):846-850. doi:10.1016/01909622(92)70119-Z

7. Park JY, Lee HS, Cho KH. Cutaneous metastasis to the scalp from squamous cell carcinoma of the cervix. Clin Exp Dermatol. 2003;28(1):28-30. doi:10.1046/ j.1365-2230.2003.01128.x

8. Agarwal U, Dahiya P, Chauhan A, Sangwan K, Purwar P. Scalp metastasis in carcinoma of the uterine cervix-a rare entity. Gynecol Oncol. 2002;87(3):310-312. doi:10.1006/gyno.2002.6829

9. Takagi H, Miura S, Matsunami K, Ikeda T, Imai A. Cervical cancer metastasis to the scalp: case report and literature review. Eur J Gynaecol Oncol. 2010;31(2):217-218.

10. Fogaça MF, Fedorciw BJ, Tahan SR, Johnson R, Federman M.Cutaneous metastasis of neuroendocrine carcinoma of uterine origin. J Cutan Pathol. 1993;20(5):455-458. doi:10.1111/j.1600-0560.1993. tb00671.x

11. Vitorino-Araujo JL, Veiga JC, Barboza VR, et al. Scalp, skull and brain metastasis of squamous cell carcinoma of the cervix--a rare entity. Br J Neurosurg. 2013;27(4):519-520. doi:10.3109/02688697.2013.764 971

12. Rosen T. Cutaneous metastases. Med Clin North Am. 1980;64(5):885-900. doi:10.1016/s00257125(16)31572-3

13. Imachi $M$, Tsukamoto $\mathrm{N}$, Kinoshita $\mathrm{S}$, Nakano $\mathrm{H}$. Skin metastasis from carcinoma of the uterine cervix. Gynecol Oncol. 1993;48(3):349-354. doi:10.1006/ gyno.1993.1061

14. Tharakaram S, Rajendran SS, Premalatha S, Yesudian P, Zahara A. Cutaneous metastasis from carcinoma cervix. Int J Dermatol. 1985;24(9):598-599. doi:10.1111/j.1365-4362.1985.tb05860.x

15. Chung JJ, Namiki T, Johnson DW. Cervical cancer metastasis to the scalp presenting as alopecia neoplastica. Int J Dermatol. 2007;46(2):188-189. doi:10.1111/j.1365-4632.2007.03183.x

16. Srivastava K, Singh S, Srivastava M, Srivastava AN. Incisional skin metastasis of a squamous cell cervical carcinoma 3.5 years after radical treatment--a case report. Int J Gynecol Cancer. 2005;15(6):1183-1186. doi:10.1111/j.1525-1438.2005.00173.x

17. Long $\mathrm{HJ}$ 3rd. Management of metastatic cervical cancer: review of the literature. J Clin Oncol. 2007;25(20):2966-2974. doi:10.1200/jco.2006.09.3781

(C) 2020 The Author(s); This is an open-access article distributed under the terms of the Creative Commons Attribution License (http:// creativecommons.org/licenses/by/4.0), which permits unrestricted use, distribution, and reproduction in any medium, provided the original work is properly cited. 